\title{
Acetylation stabilizes chloride intracellular channel 1 that exerts a tumor-promoting role by activation of nuclear factor kappa B in cervical cancer
}

\section{Wanyue Wang}

Qiqihar Medical University

Xin Li

Tumor Hospital of Harbin Medical University

YeXu

Tumor Hospital of Harbin Medical University

Weikang Guo

Tumor Hospital of Harbin Medical University

Hui Yu

Tumor Hospital of Harbin Medical University

\section{Lu Zhang}

Tumor Hospital of Harbin Medical University

Yaoxian Wang ( $\sim$ wyxxs012@126.com )

Cancer Hospital of Harbin Medical University

Xiuwei Chen

Tumor Hospital of Harbin Medical University

\section{Research}

Keywords: cervical cancer, chloride intracellular channel 1, nuclear factor kappa B, acetylation, acetylation

Posted Date: May 7th, 2020

DOI: https://doi.org/10.21203/rs.3.rs-26424/v1

License: (1) (1) This work is licensed under a Creative Commons Attribution 4.0 International License. Read Full License 


\section{Abstract}

Background: Cervical cancer remains a major cause of cancer-related death in women, especially in developing countries. Previously, we identified that acetylation levels of chloride intracellular channel 1 (CLIC1) at lysine 131 were increased in cervical cancer tissues by using the label-free proteomics approach. The aim of this study was further to determine the role of CLIC1 expression and its acetylation in cervical cancer.

Methods: CLIC1 expression and its implications on prognosis in cervical cancer were analyzed using Gene Expression Profiling Interactive Analysis (GEPIA) database (gepia.cancer-pku.cn). The effect of CLIC1 on cells was evaluated by Cell Counting Kit (CCK)-8, flow cytometry, wound healing assay, transwell assay, western blotting, and co-immunoprecipitation (Co-IP). In vivo tumor growth was assessed in mouse xenograft models.

Results: We found that CLIC1 expression was increased in cervical cancer tissues and cells. The knockdown of CLIC1 significantly reduced cell proliferation, migration, invasion, and in vivo tumorigenesis of cervical cancer cells. Molecularly, nuclear factor kappa B (NF-kB) activity was positively regulated by CLIC1. Pyrrolidine dithiocarbamate (PDTC), an inhibitor of NF-KB activation, attenuated the tumor-promoting effect of CLIC1. Moreover, CLIC1 acetylation at K131 was upregulated in cervical cancer, which stabilized CLIC1 by inhibiting ubiquitylation. The substitution of K131 inhibited CLIC1 ubiquitylation and promoted cell proliferation, migration, and invasion, and tumor growth. In addition, we identified that acetyltransferase HAT1 was responsible for CLIC1 acetylation at K131.

Conclusion: This finding shows that CLIC1 is a tumor promoter in cervical cancer, indicating a potential strategy to treat cervical cancer by regulating CLIC1 expression or acetylation.

\section{Introduction}

Cervical cancer ranks as the fourth for both incidence and mortality in female malignancy worldwide, with an estimated 569847 new cases and 311365 deaths reported worldwide in 2008 [1]. The mortality of cervical cancer in low-income and middle-income countries is 18 times higher than that in developed countries [2]. Although the improvement in therapeutic strategies in the past few decades, the prognosis of patients with advanced or recurrent metastatic cervical cancer remains poor [3]. Thus, it is necessary for further understanding of the molecular mechanisms involved in cervical cancer development.

Chloride intracellular channel 1 (CLIC1), a member of the p64 family, is a small protein composed of 241 amino acids, with a molecular weight of $27 \mathrm{kDa}$ [4]. CLIC1 exerts crucial functions in multiple physiological and pathological processes, such as

osteoblastic differentiation [5], neurotoxicity [6], neurite elongation [7]. Moreover, increasing evidences show that CLIC1 is involved in tumor progression. The expression of CLIC1 is upregulated in a variety of different cancers, such as oral squamous cell carcinoma [8], epithelial ovarian cancer [9], gastric cancer 
[10], gallbladder cancer [11], pancreatic cancer [12], and gliomas [13], and increased CLIC1 levels are associated with a poor prognosis in these patients. Moreover, knockout of CLIC1 inhibits medulloblastoma growth in xenograft and genetically engineered mouse models [14]. Overexpression of CLIC1 promotes the growth of oral squamous cell carcinoma cells by activating integrins/ERK pathways [15]. The silencing of CLIC1 represses cell viability and invasive potency of hepatocarcinoma cells by regulating maspin expression [16]. However, the function and molecular mechanism of CLIC1 in cervical cancer are unclear.

Lysine acetylation is a common posttranslational modification (PTM) regulating protein stability, subcellular localization, and function [17]. Acetylation of histone and nonhistone was reported to be involved in many diseases, including aging-associated chronic inflammation and insulin resistance [18], cancer [19-21], colitis [22], and Alzheimer's disease [23]. In previous work, we analyzed the lysine acetylproteome in three primary cervical cancer tissues and corresponding adjacent normal tissues by using the label-free proteomics approach and found that acetylation levels of CLIC1 at lysine 131 were significantly increased in tumor tissues. Hence, we deduce that CLIC1 acetylation at lysine 131 may play a crucial role in cervical cancer tumorigenesis.

In the present study, we demonstrated that both levels of CLIC1 and its acetylation at lysine 131 were upregulated in cervical cancer. CLIC1 could promote cell proliferation, migration, invasion, and in vivo tumorigenesis by regulating nuclear factor kappa B (NF-KB) activity. Furthermore, Acetylation of CLIC1 at lysine 131 inhibited its ubiquitination and degradation, thus leading to an increase of CLIC1 protein. Moreover, we showed that acetyltransferase 1 (HAT1) acetylated CLIC1 at lysine 131 and increased protein stability. Our observations reveal a novel function and mechanism of CLIC1 upregulation in cervical cancer.

\section{Materials And Methods}

\section{Clinical samples and animals}

A total of 30 paired cervical cancer tissues and corresponding adjacent tissues were obtained from Harbin Medical University Cancer Hospital. Each patient signed informed consent prior to participating in the study. All procedures involving human subjects were approved by the Ethics Committee of the Harbin Medical University and were in accordance with the Declaration of Helsinki.

Sixty BALB/c nude mice were purchased from Beijing Huafukang Bioscience Co. Inc. (Beijing, China) and housed in cages with a $12 \mathrm{~h}$ light/dark cycle and food and water were freely available. All procedures involving animal experiments were approved by the Animal Care and Use Committee of Harbin Medical University and were in compliance with the Guide for the Care and Use of Laboratory Animals.

\section{Cells and cell culture}


Human cervical epithelial cells (HCerEpiC) and cervical cancer cells (Hela, Siha, C-33A, and CaSki) were purchased from Procell (Wuhan, China). HCerEpiC, Siha, Hela, and C-33a were cultured in minimal essential medium (Gibco, Grand Island, NY, USA) containing 10\% fetal bovine serum (FBS; Hyclone, Logan, UT, USA). Caski was cultured in Roswell Park Memorial Institute 1640 medium (Gibco) plus 10\% FBS (Hyclone). All cells were maintained in an incubator set to $37^{\circ} \mathrm{C}$ and $5 \% \mathrm{CO} 2$. Pyrrolidine dithiocarbamate (PDTC), an inhibitor of NF-KB activation, was obtained from Aladdin Reagent Company (Shanghai, China). After transfection for 24 h, $200 \mu \mathrm{mol} / \mathrm{L}$ PDTC was added to cells for $24 \mathrm{~h}$.

\section{Transient transfection and stable cell lines generation}

Specific small interfering RNAs (siRNAs) against CLIC1 and HAT1 were obtained from JTS Scientific (Hubei, Wuhan, China). Recombinant plasmids expressing CLIC1, P300/CBP-associated factor (PCAF), E1A binding protein p300 (P300), CREB-binding protein (CBP), General Control Of Amino Acid Synthesis Protein 5-Like 2 (GCN5), Tat Interacting Protein, 60kDa (Tip60), and HAT1 were purchased from GenScript (Piscataway, NJ, USA). Recombinant plasmids expressing Flag-tagged CLIC1 or Flag-tagged mutant CLIC1 (lysine-to-arginine at position 131, CLIC1 K131R) were obtained from ViGene Biosciences (Shandong, China). They were transiently transfected into Hela or Siha cells for $48 \mathrm{~h}$ (except for cell proliferation assay) using Lipofectamine 2000 (Invitrogen, Carlsbad, CA, USA). For in vivo experiments, cells with stable overexpression or knockdown of CLIC1 were selected and used.

\section{Protein separation and western blot analysis}

Total protein was extracted from cells or tumor tissues using RIPA lysis buffer (Solarbio, Beijing, China). Nuclear extracts were obtained from cells with a Nuclear Protein Extraction Kit (Solarbio). Protein concentration was determined with a BCA Protein Assay kit (Solarbio). The samples were subjected to sodium dodecyl sulfate-polyacrylamide gel electrophoresis (SDS-PAGE), transferred onto PVDF membranes, and blocked with $5 \%$ skimmed milk. Afterward, the membranes were incubated with primary antibodies against CLIC1 (1:1000, Abcam, Cambridge, MA, USA), p-p65 (1:500, CST, Danvers, MA, USA), p65 (1:1000, CST), p-IkB (1:500, CST), IkB (1:1000, CST), PCAF (1:3000, CST), P300 (1:3000, CST), CBP (1:3000, CST), GCN5 (1:3000, ABclonal, Boston, MA, USA), Tip60 (1:3000, Abclonal), HAT1 (1:3000, Abclonal), Histone H3 (1:5000, Gene Tex, California, USA), GAPDH (1:10000, Proteintech, Wuhan, China), Ubiquitin (1:1000, Abcam), and Flag (1:1000, KangWei, Beijing, China) overnight $4^{\circ} \mathrm{C}$. Protein bands were visualized by an ECL detection method (Solarbio) and quantitated by densitometry.

\section{Cell proliferation assay}

Cell proliferation ability was evaluated by Cell Counting Kit (CCK)-8 assay. Briefly, cells at a density of $4 \times 10^{3}$ were seeded in 96-well plates in quintuplicate. After treatment, the plates were incubated for 0,24 , $48,72,96$, or $120 \mathrm{~h}$ at $37^{\circ} \mathrm{C}$. Ten microliters of CCK-8 solution then was added to each well at indicated time points for $2 \mathrm{~h}$. The absorbance value of each well was determined at $450 \mathrm{~nm}$ using a microplate reader (BioTek, Winooski, VT, USA). 


\section{Cell apoptosis assay}

Cell apoptosis was assessed by flow cytometry after annexin V-FITC and propidium iodide (PI) staining (Beyotime Institute of Biotechnology, Shanghai, China), according to the manufacturer's instruction.

\section{Xenograft assay}

Mice were randomly divided into 12 groups $(n=6)$. Both Siha and Hela cells with stable expression of control shRNA, CLIC1 shRNA1, CLIC1 shRNA2, CLIC1 WT CLIC1 K131R were subcutaneously injected into BALB/c nude mice. Tumor volume was measured every two days. Nineteen days later, mice were sacrificed, and the tumor tissues were collected for the following western blot analysis.

\section{Cell migration assay}

Cell migration was determined by the wound healing assay. In short, the cells were grown to confluence, and $1 \mu \mathrm{g} / \mathrm{mL}$ mitomycin $\mathrm{C}$ was added to induce growth arrest. Then $200 \mu \mathrm{L}$ of pipette tip was used to create a scratch in the monolayer, after which the plate was washed once and replaced with the desired medium. Images were captured with an inverted phase-contrast microscope (Olympus, Tokyo, Japan).

\section{Cell invasion assay}

Cell invasion was evaluated by the transwell assay. In brief, cells at a density of $1.5 \times 10^{4}$ were seeded into transwell chambers (Corning Inc., Corning, NY, USA) coated with Matrigel. The bottom chamber was filled with $800 \mu \mathrm{L}$ of medium containing $10 \% \mathrm{FBS}$. After $48 \mathrm{~h}$, cells were immobilized with $4 \%$ paraformaldehyde, stained with $0.4 \%$ crystal violet, and pictured and counted using a microscope (Olympus).

\section{Co-immunoprecipitation (co-IP) assay}

Total protein was isolated and quantified as described above. The equal amounts of cell lysates were subjected to incubation with specific primary antibodies overnight at $4^{\circ} \mathrm{C}$. Afterward, $60 \mu \mathrm{L}$ of protein $\mathrm{A} / \mathrm{G}$ agarose was added for $2 \mathrm{~h}$ at $4^{\circ} \mathrm{C}$. The immunoprecipitated complex was pelleted by centrifuging and analyzed by western blotting with indicated antibodies.

\section{Statistical analysis}

Statistical analyses were performed using GraphPad Prism 7 (La Jolla, CA, USA). The data are expressed as mean $\pm S D$ of three independent experiments for in vitro and six animals in each group for in vivo studies. Student's t-test was used to analyze experimental data between two groups, and one- or two-way ANOVA was used to test the difference between more than two experimental groups. Differences were considered statistically significant at $* \mathrm{p}<0.05$.

\section{Results}




\section{CLIC1 expression is frequently upregulated in cervical cancer}

The expression levels of CLIC1 in 30 pairs of cervical cancer and matched normal cervix tissue samples were determined by western blot analysis. The results showed that CLIC1 expression in tumor tissues was increased in 21 of 30 cases, Fig. 1A showed the representative results from eight individual patients. Moreover, the Gene Expression Profiling Interactive Analysis (GEPIA) database (http://gepia.cancerpku.cn/), a web-based tool to deliver fast and customizable functionalities based on TCGA and GTEx data, indicated that CLIC1 mRNA levels were significantly up-regulated in cervical cancer tissues compared with normal tissues (Fig. 1B), and that cervical cancer patient with high CLIC1 expression tend to have shorter overall survival (Additional file 1). Furthermore, by western blot analyses, we found that CLIC1 expression was overexpressed in human cervical carcinoma cell lines (Hela, Siha, C-33A, and CaSki) compared to human cervical epithelial cells (HcerEpic).

\section{CLIC1 knockdown inhibits cervical cancer malignant phenotypes}

Subsequently, the function of CLIC1 in cervical cancer cells was investigated. Two siRNAs targeting CLIC1 were used to silence gene expression in Siha and Hela cells. The knockdown efficiency was validated by real-time PCR (Fig. 2A). Furthermore, the CCK-8 assay showed that cell proliferation ability was markedly inhibited in cervical cancer cells transfected with CLIC1 siRNAs (Fig. 2B). The apoptosis was determined using flow cytometry with annexin V-FITC/PI staining, the results showed that apoptotic cells were significantly increased in CLIC1-silenced Siha and Hela cells (Fig. 2C). The knockdown of CLIC1 suppressed the tumorigenesis of cervical cells in mouse xenograft models in vivo (Fig. 2D and E). Moreover, the wound healing assay demonstrated that cell migration activity was apparently attenuated following downregulation of CLIC1 (Fig. 3A). Transwell assay indicated that the silencing of CLIC1 inhibited cell invasiveness (Fig. 3B).

\section{CLIC1 knockdown inactivates NF-kB pathway}

The effect of CLIC1 knockdown on NF-kB, a critical regulator of oncogenic processes, was further investigated. As shown in Fig. 4A and B, the expression levels of P-p65, P-IKBa, nuclear p65 were reduced, while IKBa was increased in Siha and Hela cells transfected with CLIC1 siRNAs. Similar results for the expression of P-p65, P-IKBa, IKBa were obtained in tumor tissues derived from Siha or Hela cells with stable knockdown of CLIC1 (Fig. 4C).

\section{CLIC1 overexpression promotes cervical cancer malignant phenotypes by}

\section{activating NF-kB pathway}

Furthermore, we explored the influence of CLIC1 upregulation on cervical cancer cells and the role of NFKB in CLIC1-mediated actions. In contrast to the effects caused by CLIC1 downregulation, overexpression of CLIC1 induced NF-KB activity (Fig. 5A and B), promoted cell proliferation (Fig. 5C), migration (Fig. 5D), and invasion (Fig. 5E). The tumor-promoting effects driven by forced overexpression of CLIC1 could be attenuated by the treatment of PDTC, an inhibitor of NF-KB activation (Fig. 5C-E). 


\section{Acetylation of CLIC1 at K131 stabilizes protein by blocking its ubiquitination}

In previous work, we found that acetylation levels of CLIC1 at lysine 131 were dramatically augmented in three primary cervical cancer tissues versus corresponding adjacent normal tissues by using the labelfree proteomics approach. In order to further validate the acetylation of this site, we generated an antibody specific to acetylated K131. As shown in Fig. 6A and B, the acetylation levels of CLIC1 at K131 were increased in cervical cancer tissues and cells used in Fig. $1 \mathrm{~A}$ and $\mathrm{C}$. Moreover, we found a significant decrease in acetylation levels of CLIC1 when K131 was mutated to arginine (CLIC1 K131R) in Hela cells (Fig. 7A). The protein stability of CLIC1 K131R was increased compared to wild type CLIC1 (CLIC1 WT) (Fig. 7A). The CLIC1 ubiquitylation levels were reduced in Hela cells expressing CLIC1 K131R (Fig. 7B). The effect of the K131R mutant of CLIC1 on cell function in vitro and in vivo was further studied. As shown in Fig. 7C-G, overexpression of CLIC1 K131R markedly promoted cell proliferation, migration, invasion, and in vivo tumorigenesis.

\section{HAT1 acetylates CLIC1}

To identify the acetyltransferase responsible for CLIC1 K131 acetylation, Hela cells were transfected with six acetyltransferases PCAF, P300, CBP, GCN5, Tip60, or HAT1. Western blot analysis showed that HAT1 overexpression upregulated K131 acetylation of CLIC1, whereas others did not (Fig. 8A). In addition, co-IP and western blotting assays confirmed the binding of HAT1 with CLIC1 (Fig. 8B). The silencing of HAT1 by two siRNAs led to a decrease in levels of CLIC1 and CLIC1 K131 acetylation (Fig. 8C).

\section{Discussion}

The tumor-promoting role of CLIC1 has been reported in a variety of tumors $[12,14,15]$. Indirectly, miR124 inhibits the proliferation, migration, and invasion of liver cancer cells by targeting CLIC1 [24]; PA28 $\beta$ enhances invasion and metastasis of gastric cancer via upregulating CLIC1 [25]. Nevertheless, whether CLIC1 plays a role in cervical cancer is not clear yet. In the present study, we observed that increased expression of CLIC1 was frequently detected in cervical cancer tissues and cells. Analysis of loss- and gain-of-function phenotypes showed that CLIC1 positively regulated cell proliferation, migration, invasion, and in vivo tumorigenesis, indicating that CLIC1 is acting as a tumor promoter in cervical cancer.

The transcription factor NF-KB was first reported in cells B cells as a nuclear factor that binds to the enhancer element of the immunoglobulin kappa light-chain [26]. The NF-kB family has five members, including p50, p52, p65 (RelA), c-Rel, and RelB, all of which contain an N-terminal Rel homology domain (RHD) that mediates DNA contact and homo- and heterodimerization [27]. In physiological conditions, NFKB homo- and heterodimers are kept inactive in the cytoplasm by the interactions with IKB inhibitors

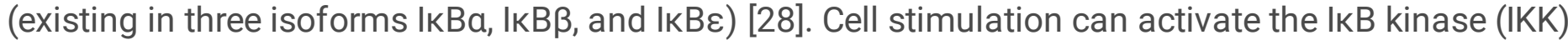
complex, which is responsible for the ІкB phosphorylation, marking it for degradation via the $\beta$-transducin repeat-containing protein ( $\beta$-TrCP)-dependent E3 ubiquitin ligase-mediated proteasomal degradation 
machinery, leading to NF-kB nuclear translocation and then activates its target genes associated with biological processes, including cancer [29]. In our study, we demonstrated that CLIC1 inhibited IкBa expression and promoted phosphorylation and translocation of p65 and p-lkBa expression. The protumor effects mediated by CLIC1 could be suppressed by NF-KB inhibitor (PDTC), suggesting the key role of the CLIC1/NF-KB axis in tumor progression.

PTM of proteins is emerging as a general regulatory mechanism in a diverse array of cellular processes, such as cell metabolism, protein degradation, protein trafficking, signal transduction, and the DNA damage response. Lysine acetylation is one of the most versatile PTMs of proteins and plays a critical role in many diseases including cancer [30]. Moreover, acetylation would prevent the ubiquitination of the same lysine residues [31]. The PTMs of proteins have been detected by a variety of experimental techniques including the mass spectrometry (MS), liquid chromatography, radioactive chemical method, and chromatin immune precipitation (ChIP) [32]. Previously, we found that lysine acetylation of CLIC1 at K131 was significantly upregulated in cervical tissues by using the label-free proteomics approach. Here, the ubiquitination levels of K131R mutant CLIC1 were decreased, leading to increased CLIC1 protein stability, and thus resulting in more aggressive cell phenotypes.

HAT1 is the first lysine acetyltransferase originally identified in yeast in 1995 [33]. Subsequently, it was suggested that HAT1 protein is cytoplasmic and involved in histone deposition [34]. In addition to acetylation of histones, HAT1 has also been shown to acetylate nonhistone proteins, such as promyelocytic leukemia zinc finger protein (PLZF) [35], and p53 [36]. In the present study, we demonstrated that HAT1 could acetylate CLIC1 at K131 and hence increase the stability of CLIC1 by inhibiting its ubiquitination. However, the deacetylase regulating CLIC1 acetylation needs to be further investigated.

In conclusion, our results suggest that CLIC1 is frequently increased and acts as a tumor promoter in cervical cancer and that acetylation of CLIC1 at k131 by HAT1 stabilizes CLIC1 protein and protects it from ubiquitination-mediated degradation. This study presents a potential strategy for the treatment of cervical cancer by regulating the expression and acetylation of CLIC1.

\section{Abbreviations}

CBP: CREB-binding protein; CCK-8: Cell Counting Kit (CCK)-8; ChIP: chromatin immune precipitation; CLIC1: chloride intracellular channel 1; Co-IP: co-immunoprecipitation; FBS: fetal bovine serum; GCN5: General Control of Amino Acid Synthesis Protein 5-Like 2; GEPIA: Gene Expression Profiling Interactive Analysis; HAT1: acetyltransferase 1; HCerEpiC: Human cervical epithelial cells; IKK: IKB kinase; MS: mass spectrometry; NF-KB: nuclear factor kappa B; P300: E1A binding protein p300; PCAF: P300/CBPassociated factor; PDTC: pyrrolidine dithiocarbamate; PI: propidium iodide; PTM: posttranslational modification; RHD: Rel homology domain; SDS-PAGE: sodium dodecyl sulfate-polyacrylamide gel electrophoresis; siRNAs: small interfering RNAs; Tip60: Tat Interacting Protein, 60 kDa; $\beta$-TrCP: $\beta$ transducin repeat-containing protein. 


\section{Declarations}

Acknowledgments

Not applicable.

\section{Authors' contributions}

WYX and CXW conceived and designed the study. WWY, LX, and XY conducted experiments. WWY, GWK, $Y H$, and $Z L$ analyzed data. WWY and LX wrote the manuscript. WYX and CXW modified the manuscript. All authors read and approved the final manuscript.

\section{Funding}

This study was supported by grants from the National Natural Science Foundation of China (No. 81772274), the Natural Science Foundation of Heilongjiang Province (No. H2017045), and the Key Project for Petrel Foundation of Harbin Medical University Cancer Hospital (No. JJZD2020-07).

\section{Availability of data and materials}

The analyzed data sets generated during the study are available from the corresponding author on reasonable request.

\section{Ethics approval and consent to participate}

The study was approved by the Ethics Committee of the Harbin Medical University and the written informed consent was obtained from all patients. All animal experiments were in line with the Guide for the Care and Use of Laboratory Animal by the National Institutes of Health.

\section{Consent for publication}

Not applicable.

\section{Competing interests}

The authors declare no competing financial interests.

\section{Author details}

${ }^{1}$ School of Basic Medical Sciences, Qiqihar Medical University, Qiqihar, Heilongjiang 161006, China.

${ }^{2}$ Department of Oncology, Harbin Medical University Cancer Hospital, No. 150 Haping Road, Nangang District, Harbin 150081, Heilongjiang Province, China. ${ }^{3}$ Department of Gynecology, Harbin Medical University Cancer Hospital, No. 150 Haping Road, Nangang District, Harbin 150081, Heilongjiang Province, China. ${ }^{4}$ Department of Cardiopulmonary Function, Harbin Medical University Cancer Hospital, No. 150 Haping Road, Nangang District, Harbin 150081, Heilongjiang Province, China. 


\section{References}

1. Bray F, Ferlay J, Soerjomataram I, Siegel RL, Torre LA, Jemal A. Global cancer statistics 2018: GLOBOCAN estimates of incidence and mortality worldwide for 36 cancers in 185 countries. CA Cancer J Clin. 2018;68(6):394-424.

2. Cohen PA, Jhingran A, Oaknin A, Denny L. Cervical cancer. Lancet. 2019;393(10167):169-82.

3. Wei R, Jiang G, Lv M, Tan S, Wang X, Zhou Y, et al. TMTP1-modified Indocyanine Green-loaded Polymeric Micelles for Targeted Imaging of Cervical Cancer and Metastasis Sentinel Lymph Node in vivo. Theranostics. 2019;9(24):7325-44.

4. Averaimo S, Milton RH, Duchen MR, Mazzanti M. Chloride intracellular channel 1 (CLIC1): Sensor and effector during oxidative stress. FEBS Lett. 2010;584(10):2076-84.

5. Yang JY, Jung JY, Cho SW, Choi HJ, Kim SW, Kim SY, et al. Chloride intracellular channel 1 regulates osteoblast differentiation. Bone. 2009;45(6):1175-85.

6. Novarino G, Fabrizi C, Tonini R, Denti MA, Malchiodi-Albedi F, Lauro GM, et al. Involvement of the intracellular ion channel CLIC1 in microglia-mediated beta-amyloid-induced neurotoxicity. J Neurosci. 2004;24(23):5322-30.

7. Averaimo S, Gritti M, Barini E, Gasparini L, Mazzanti M. CLIC1 functional expression is required for cAMP-induced neurite elongation in post-natal mouse retinal ganglion cells. J Neurochem. 2014;131(4):444-56.

8. Xu Y, Xu J, Feng J, Li J, Jiang C, Li X, et al. Expression of CLIC1 as a potential biomarker for oral squamous cell carcinoma: a preliminary study. Onco Targets Ther. 2018;11:8073-81.

9. Yu W, Cui R, Qu H, Liu C, Deng H, Zhang Z. Expression and prognostic value of CLIC1 in epithelial ovarian cancer. Exp Ther Med. 2018;15(6):4943-9.

10. Chen CD, Wang CS, Huang YH, Chien KY, Liang Y, Chen WJ, et al. Overexpression of CLIC1 in human gastric carcinoma and its clinicopathological significance. Proteomics. 2007;7(1):155-67.

11. Ding Q, Li M, Wu X, Zhang L, Wu W, Ding Q, et al. CLIC1 overexpression is associated with poor prognosis in gallbladder cancer. Tumour Biol. 2015;36(1):193-8.

12. Lu J, Dong Q, Zhang B, Wang X, Ye B, Zhang F, et al. Chloride intracellular channel 1 (CLIC1) is activated and functions as an oncogene in pancreatic cancer. Med Oncol. 2015;32(6):616.

13. Wang L, He S, Tu Y, Ji P, Zong J, Zhang J, et al. Elevated expression of chloride intracellular channel 1 is correlated with poor prognosis in human gliomas. J Exp Clin Cancer Res. 2012;31:44.

14. Francisco MA, Wanggou S, Fan JJ, Dong W, Chen X, Momin A, et al. Chloride intracellular channel 1 cooperates with potassium channel EAG2 to promote medulloblastoma growth. J Exp Med. 2020;217(5).

15. Feng J, Xu J, Xu Y, Xiong J, Xiao T, Jiang C, et al. CLIC1 promotes the progression of oral squamous cell carcinoma via integrins/ERK pathways. Am J Transl Res. 2019;11(2):557-71.

16. Wei X, Li J, Xie H, Wang H, Wang J, Zhang X, et al. Chloride intracellular channel 1 participates in migration and invasion of hepatocellular carcinoma by targeting maspin. J Gastroenterol Hepatol. 
2015;30(1):208-16.

17. Verdin E, Ott M. 50 years of protein acetylation: from gene regulation to epigenetics, metabolism and beyond. Nat Rev Mol Cell Biol. 2015;16(4):258-64.

18. He M, Chiang HH, Luo H, Zheng Z, Qiao Q, Wang L, et al. An Acetylation Switch of the NLRP3 Inflammasome Regulates Aging-Associated Chronic Inflammation and Insulin Resistance. Cell Metab. 2020;31(3):580-91. e5.

19. Sun RC, Dukhande VV, Zhou Z, Young LEA, Emanuelle S, Brainson CF, et al. Nuclear Glycogenolysis Modulates Histone Acetylation in Human Non-Small Cell Lung Cancers. Cell Metab. 2019;30(5):90316. e7.

20. Feng $X$, Zhang H, Meng L, Song H, Zhou Q, Qu C, et al. Hypoxia-induced acetylation of PAK1 enhances autophagy and promotes brain tumorigenesis via phosphorylating ATG5. Autophagy. 2020:1-20.

21. Wu Y, Wang X, Xu F, Zhang L, Wang T, Fu X, et al. The regulation of acetylation and stability of HMGA2 via the HBXIP-activated Akt-PCAF pathway in promotion of esophageal squamous cell carcinoma growth. Nucleic Acids Res. 2020.

22. Li C, Chen Y, Zhu H, Zhang X, Han L, Zhao Z, et al. Inhibition of histone deacetylation by MS-275 alleviates colitis by activating the vitamin D receptor. J Crohns Colitis. 2020.

23. Choi H, Kim HJ, Yang J, Chae S, Lee W, Chung S, et al. Acetylation changes tau interactome to degrade tau in Alzheimer's disease animal and organoid models. Aging Cell. 2020;19(1):e13081.

24. Yue X, Cui Y, You Q, Lu Y, Zhang J. MicroRNA124 negatively regulates chloride intracellular channel 1 to suppress the migration and invasion of liver cancer cells. Oncol Rep. 2019;42(4):1380-90.

25. Zheng DL, Huang QL, Zhou F, Huang QJ, Lin JY, Lin X. PA28beta regulates cell invasion of gastric cancer via modulating the expression of chloride intracellular channel 1. J Cell Biochem. 2012;113(5):1537-46.

26. Sen R, Baltimore D. Multiple nuclear factors interact with the immunoglobulin enhancer sequences. Cell. 1986;46(5):705-16.

27. Tilborghs S, Corthouts J, Verhoeven Y, Arias D, Rolfo C, Trinh XB, et al. The role of Nuclear Factorkappa B signaling in human cervical cancer. Crit Rev Oncol Hematol. 2017;120:141-50.

28. Kearns JD, Basak S, Werner SL, Huang CS, Hoffmann A. IkappaBepsilon provides negative feedback to control NF-kappaB oscillations, signaling dynamics, and inflammatory gene expression. J Cell Biol. 2006;173(5):659-64.

29. Xia Y, Shen S, Verma IM. NF-kappaB, an active player in human cancers. Cancer Immunol Res. 2014;2(9):823-30.

30. Kaypee S, Sudarshan D, Shanmugam MK, Mukherjee D, Sethi G, Kundu TK. Aberrant lysine acetylation in tumorigenesis: Implications in the development of therapeutics. Pharmacol Ther. 2016;162:98-119. 
31. Lin R, Tao R, Gao X, Li T, Zhou X, Guan KL, et al. Acetylation stabilizes ATP-citrate lyase to promote lipid biosynthesis and tumor growth. Mol Cell. 2013;51(4):506-18.

32. He W, Wei L, Zou Q. Research progress in protein posttranslational modification site prediction. Brief Funct Genomics. 2018;18(4):220-9.

33. Kleff S, Andrulis ED, Anderson CW, Sternglanz R. Identification of a gene encoding a yeast histone $\mathrm{H} 4$ acetyltransferase. J Biol Chem. 1995;270(42):24674-7.

34. Parthun MR, Widom J, Gottschling DE. The major cytoplasmic histone acetyltransferase in yeast: links to chromatin replication and histone metabolism. Cell. 1996;87(1):85-94.

35. Sadler AJ, Suliman BA, Yu L, Yuan X, Wang D, Irving AT, et al. The acetyltransferase HAT1 moderates the NF-kappaB response by regulating the transcription factor PLZF. Nat Commun. 2015;6:6795.

36. Agudelo Garcia PA, Nagarajan P, Parthun MR. Hat1-Dependent Lysine Acetylation Targets Diverse Cellular Functions. J Proteome Res. 2020;19(4):1663-73.

\section{Figures}

A
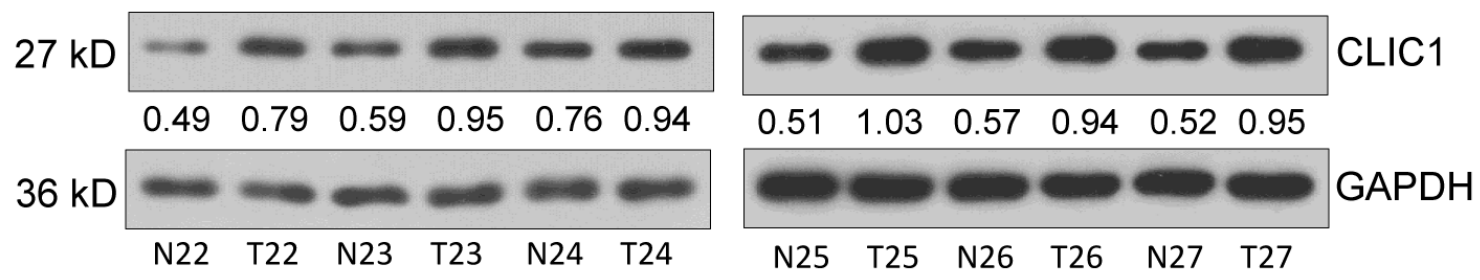

B

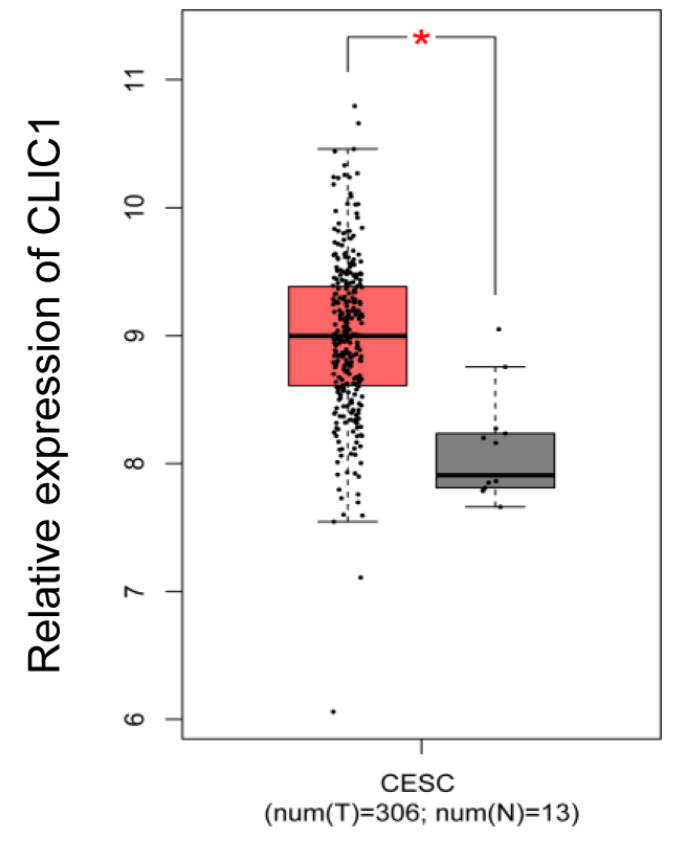

C
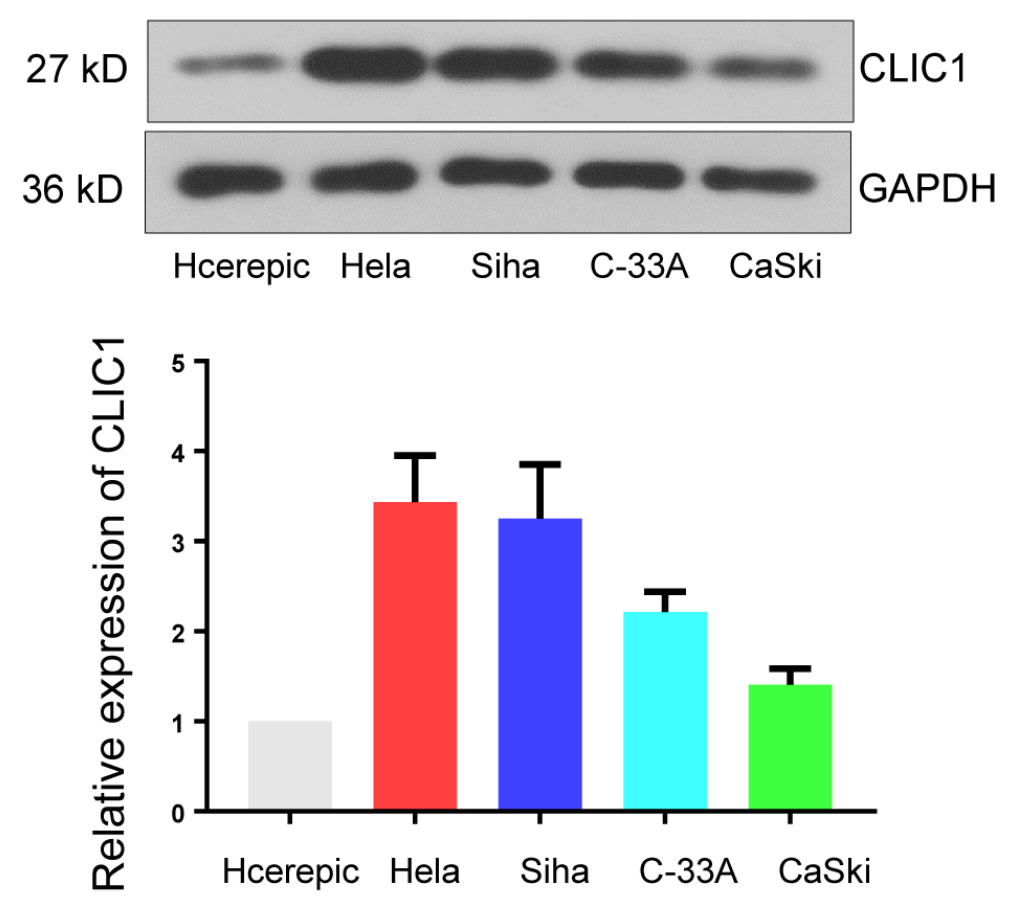
Figure 1

CLIC1 expression was increased in cervical cancer tissues and cells. (A) Western blot was employed to determine the expression of CLIC1 in 30 pairs of cervical tumor tissues and the corresponding normal tissues. (B) The mRNA expression level of CLIC1 between cervical cancer tissues and normal tissues from the TCGA and GTEx databases were assessed by the GEPIA web tool. (C) The expression of CLIC1 in human cervical epithelial (HcerEpic) cells and human cervical carcinoma cell lines (Hela, Siha, C-33A, and CaSki). Error bars in Figure $1 \mathrm{C}$ represent the SD of three independently prepared samples. ${ }^{*} \mathrm{P}<0.05$
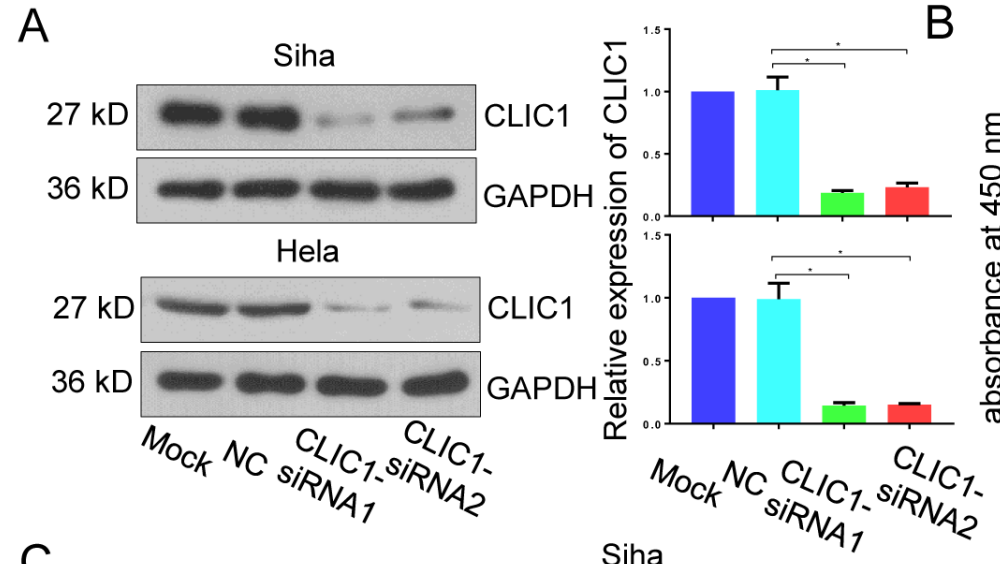

Siha

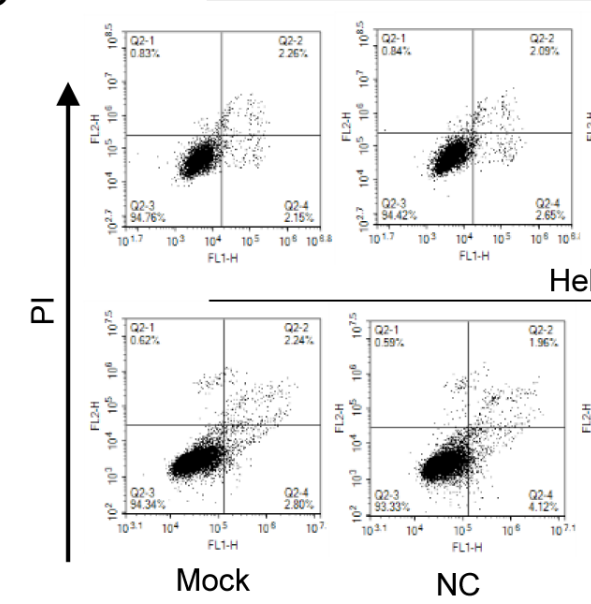

Mock

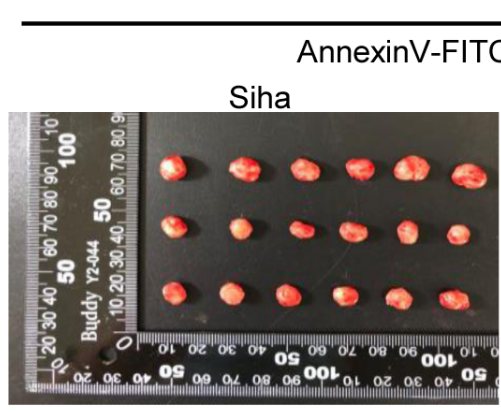

Hela

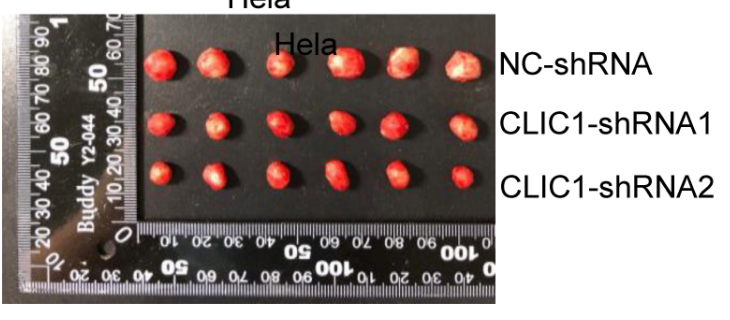

E
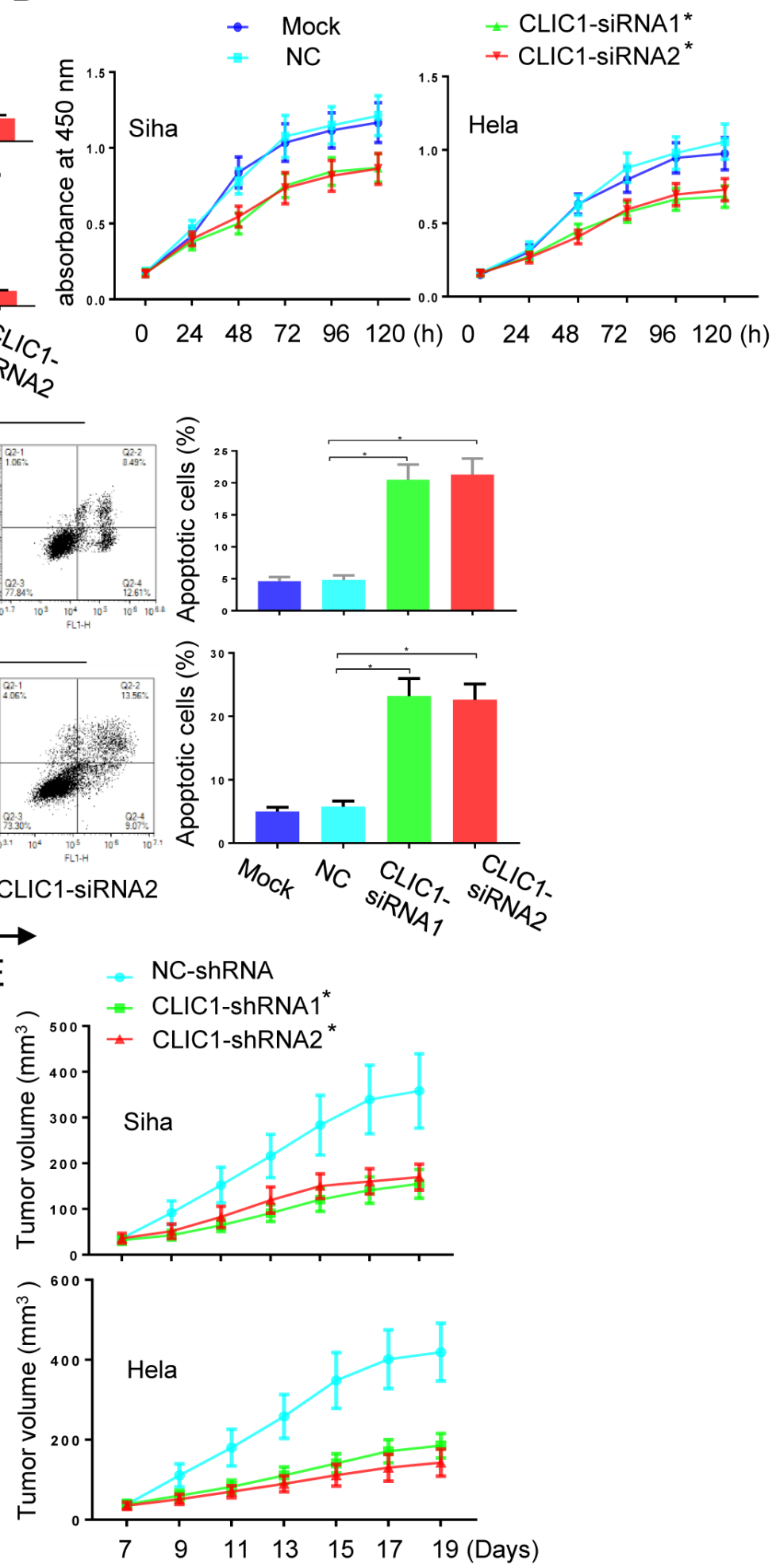

Page $13 / 20$ 


\section{Figure 2}

CLIC1 knockdown inhibited proliferation and promoted apoptosis of cervical cancer cells. Siha and Hela cells were transfected with two siRNAs targeting different regions of CLIC1 mRNA. (A) Knockdown efficiency of two independent siRNAs targeting CLIC1 determined by western blot analysis. (B) CCK-8 assay was used to evaluate the proliferation ability. (C) The cell apoptosis was determined by flow cytometry using Annexin V-FITC/PI staining. Siha and Hela cells with stably silenced expression of CLIC1 were constructed and inoculated in the subcutaneous tissue of nude mice. (D) Photographs of xenograft tumors excised from the nude mice. (F) Tumor growth curves. Error bars represent the SD of three independently prepared samples. $\mathrm{n}=6$ per group for animal studies $* \mathrm{P}<0.05$

A
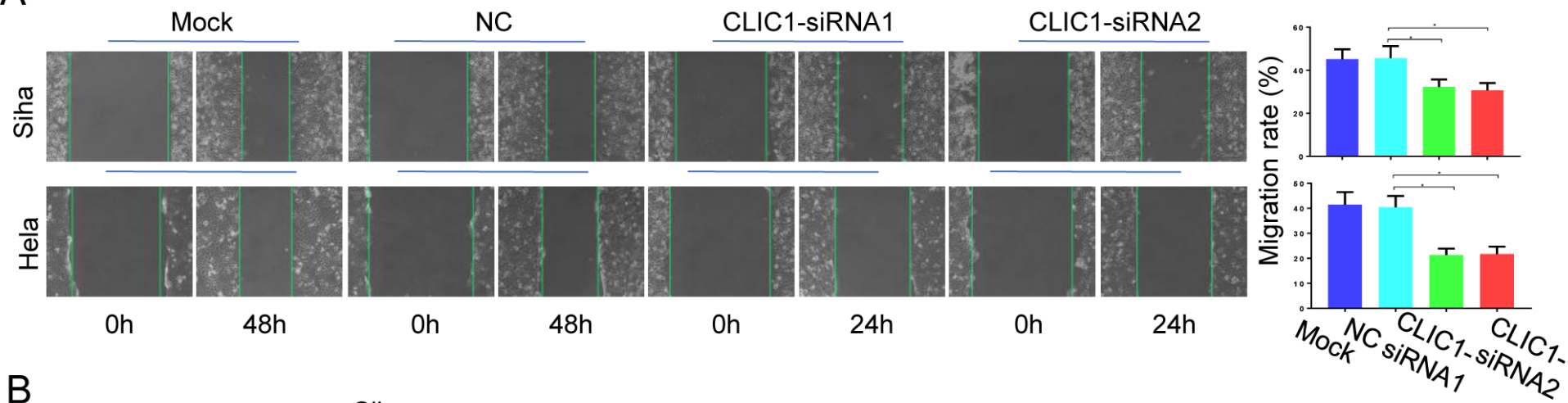

B
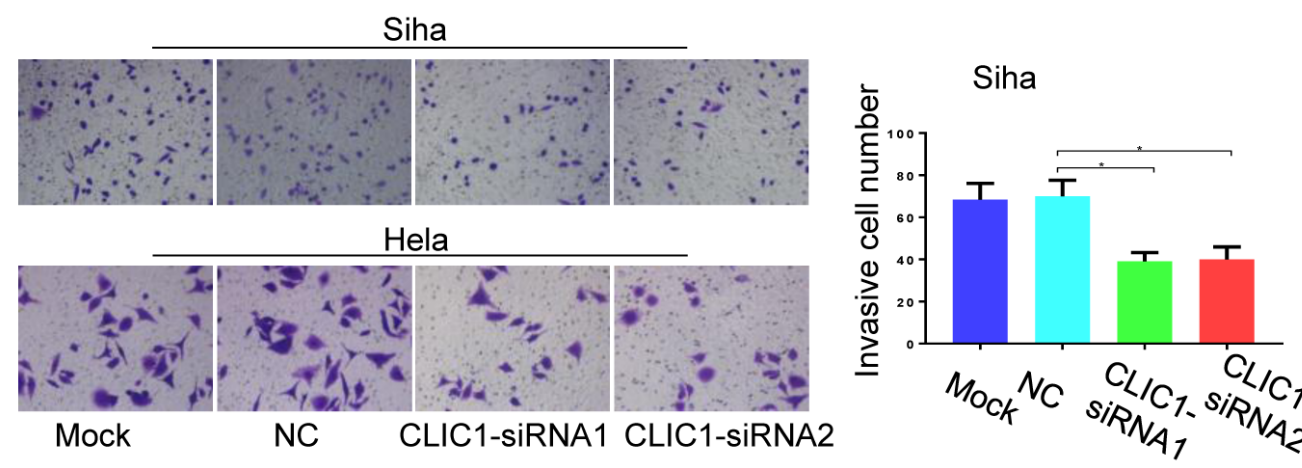

Hela

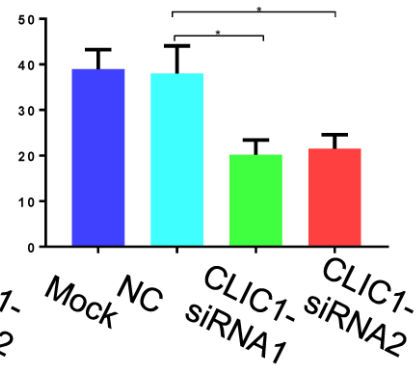

\section{Figure 3}

CLIC1 knockdown inhibited migration and invasion of cervical cancer cells. (A) An in vitro scratch wound healing assay was used to determine the migration capacity of Siha and Hela cells transfected with CLIC1 siRNAs. (B) Transwell assay was applied to study the invasion ability of cervical cancer cells with or without CLIC1 silencing. Error bars represent the SD of three independently prepared samples. ${ }^{*} \mathrm{P}<$ 0.05 


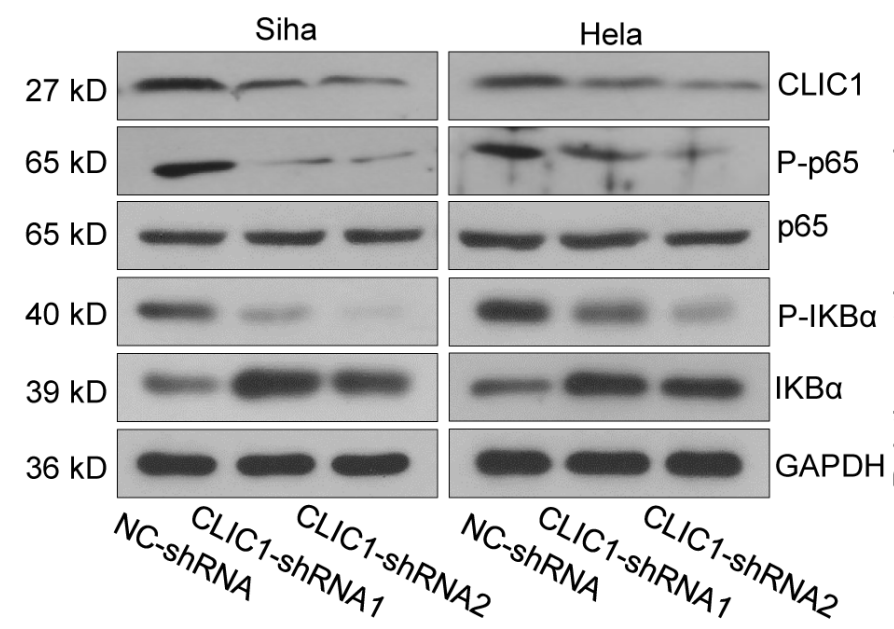

B

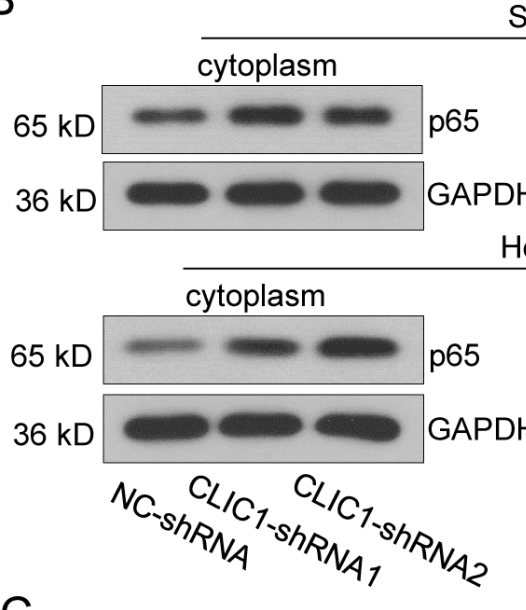

C

Siha
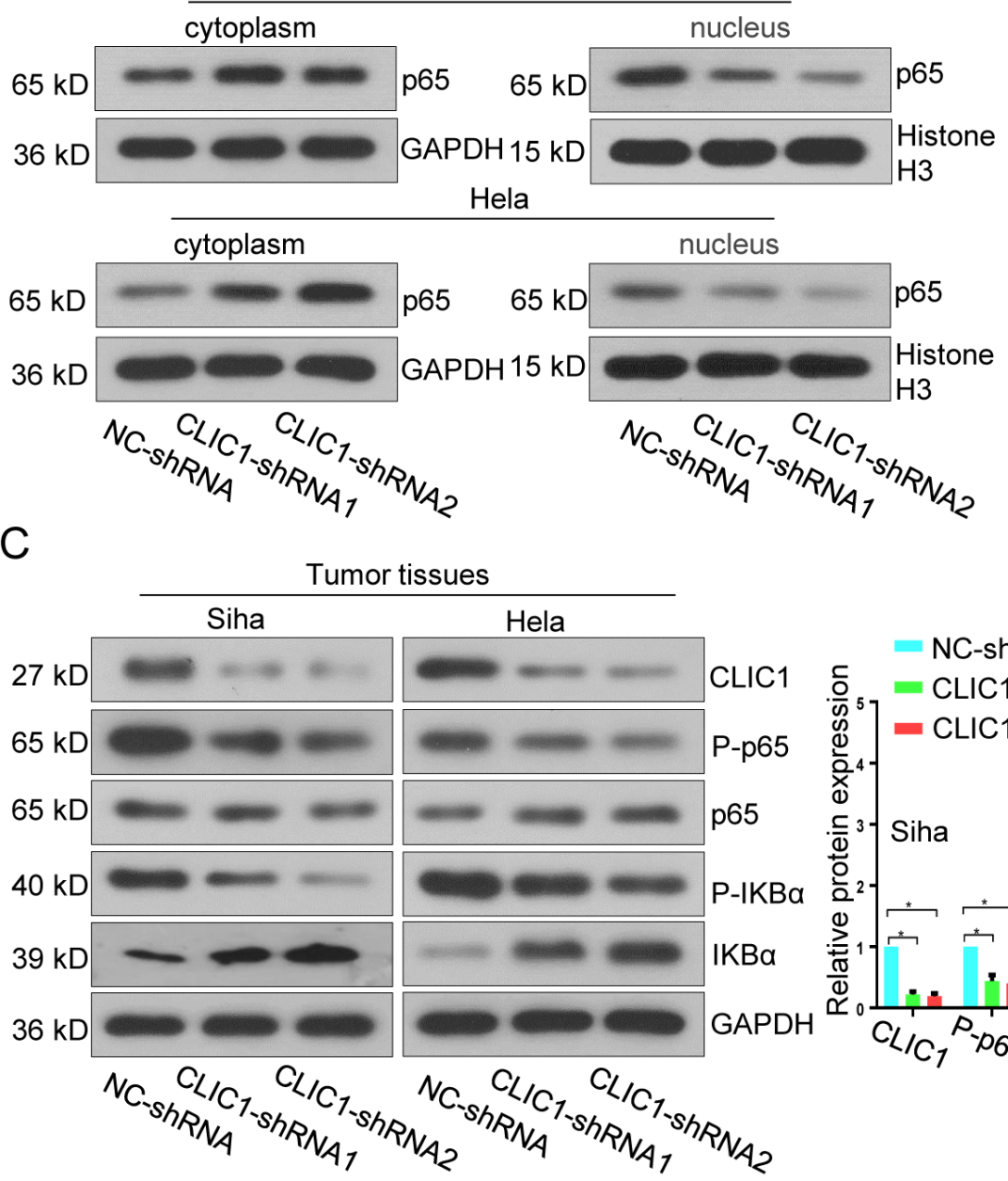

\section{Figure 4}

CLIC1 knockdown suppressed NF-kB activation in cervical cancer cells. (A) The expression levels of CLIC1, P-p65, p65, P-IKBa, and IKBa in cervical cancer cells with stably silenced expression of CLIC1 were determined by western blot assay. (B) The expression of p65 in cytoplasmic and nuclear fractions of CLIC1-silenced cervical cancer cells was evaluated by western blot assay. (C) The expression levels of CLIC1, P-p65, p65, P-IKBa, and IKBa in tumor tissues originating from cervical cancer cells with the 
downregulation of CLIC1 were determined by western blot analysis. Error bars represent the SD of three independently prepared samples. ${ }^{*} \mathrm{P}<0.05$
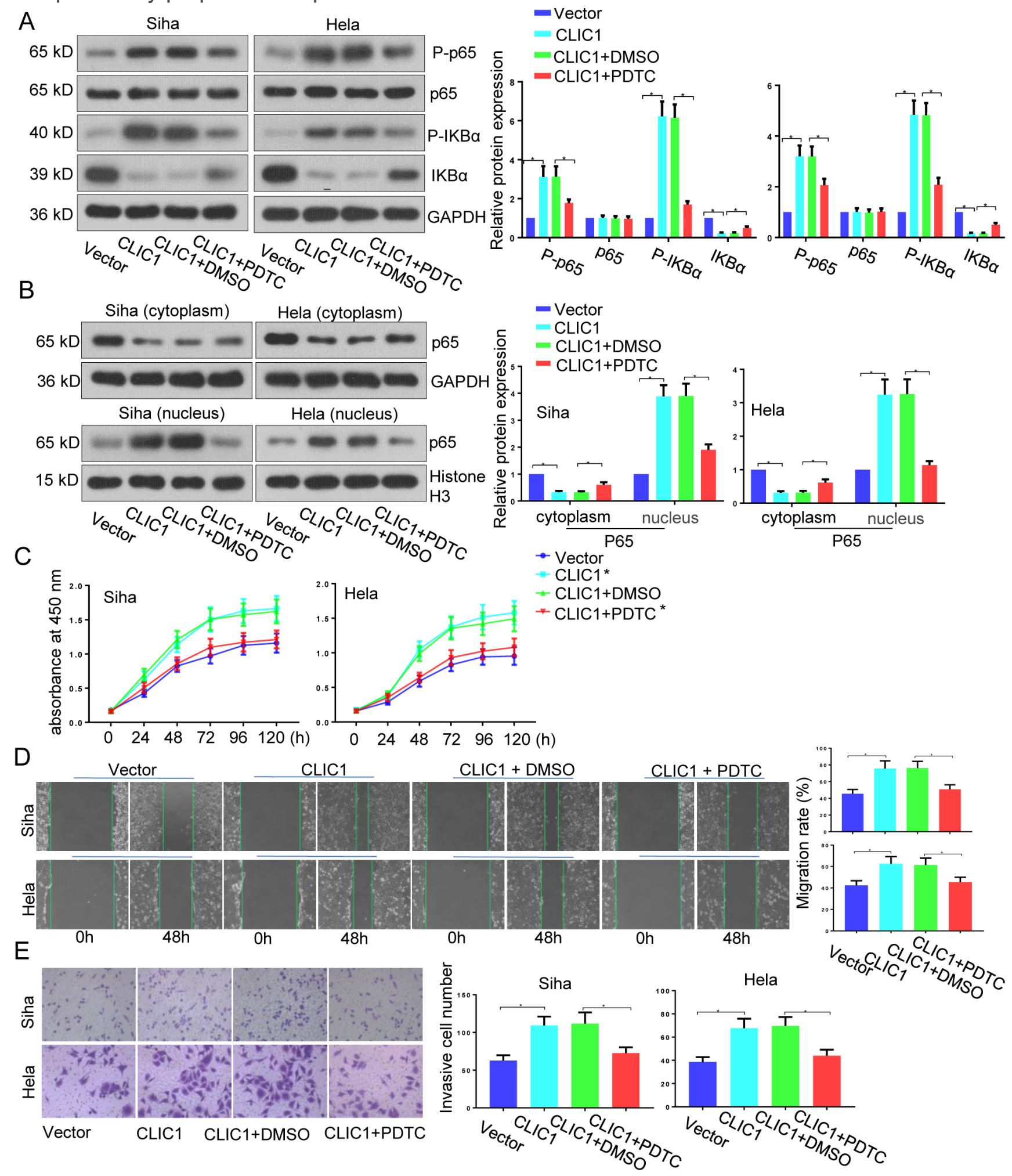

Figure 5

CLIC1 overexpression promoted the growth of cervical cancer cells by activating NF-KB. Siha and Hela cells were transfected with CLIC1-overexpressing plasmid for $24 \mathrm{~h}$, followed by treatment with PDTC. (A) The expression levels of CLIC1, P-p65, p65, P-IKBa, and IKBa were evaluated by western blot analysis. (B) 
The expression of p65 in cytoplasmic and nuclear fractions was determined by western blotting. (C) CCK8 assay was performed to assess the cell proliferation ability. (D) Cell migration ability was analyzed by scratch wound healing assay. (E) Cell invasive capability was determined by transwell assay. Error bars represent the SD of three independently prepared samples. ${ }^{*} \mathrm{P}<0.05$

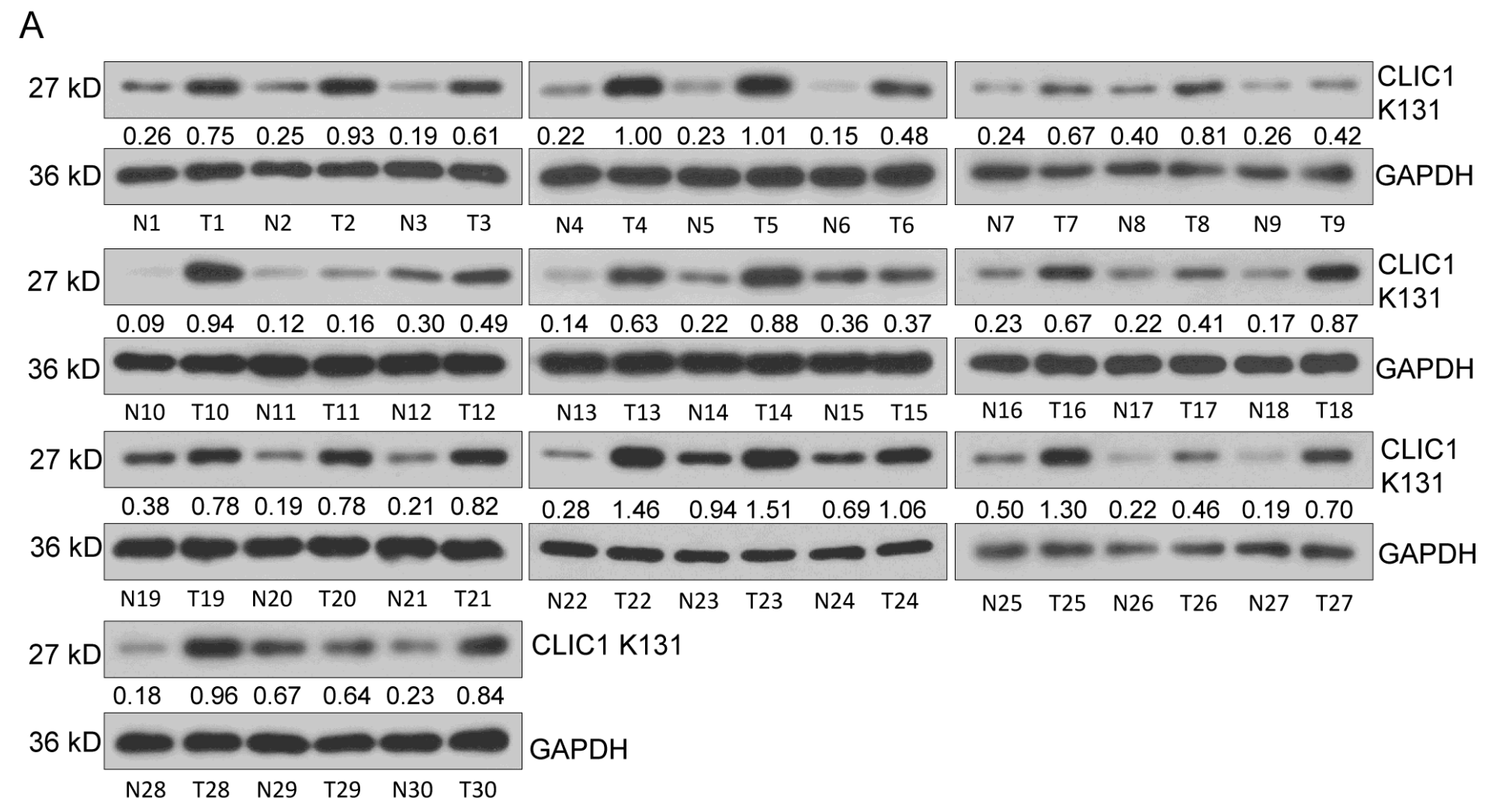

B
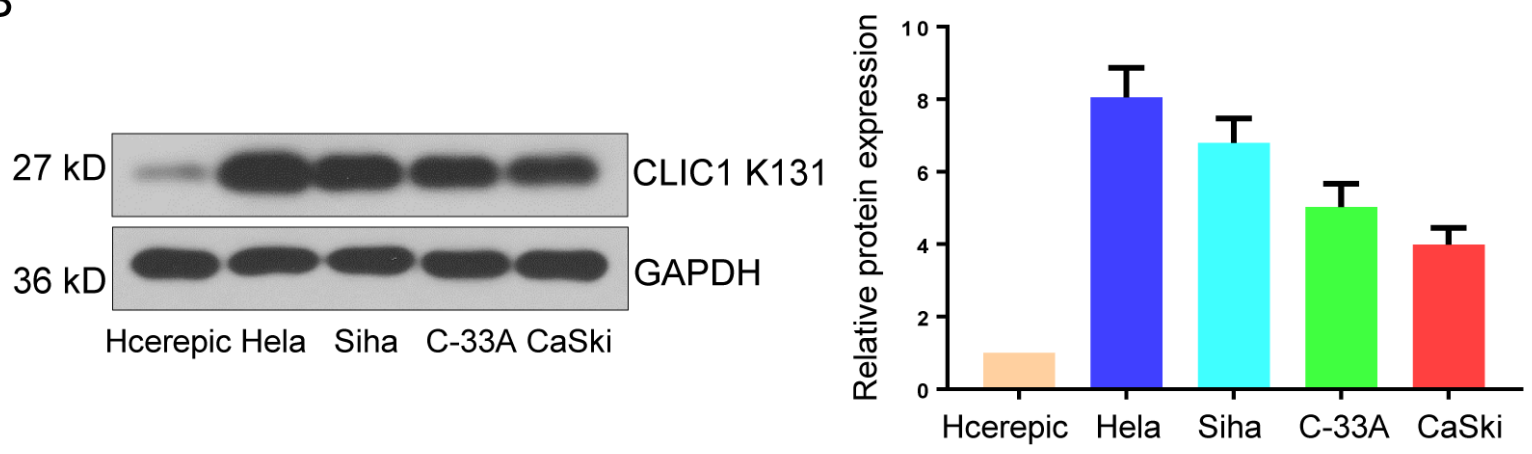

Figure 6

Acetylation of CLIC1 at K131 was increased in cervical cancer. (A) The acetylation levels of CLIC1 at K131 in 30 pairs of cervical tumor tissues and the corresponding normal tissues used in Figure 1 were examined by western blot analysis. (B) The acetylation levels of CLIC1 at K131 in human cervical epithelial (HcerEpic) cells and human cervical carcinoma cell lines (Hela, Siha, C-33A, and CaSki) were determined by western blot assay. Error bars represent the SD of three independently prepared samples. $\star P<0.05$ 
A

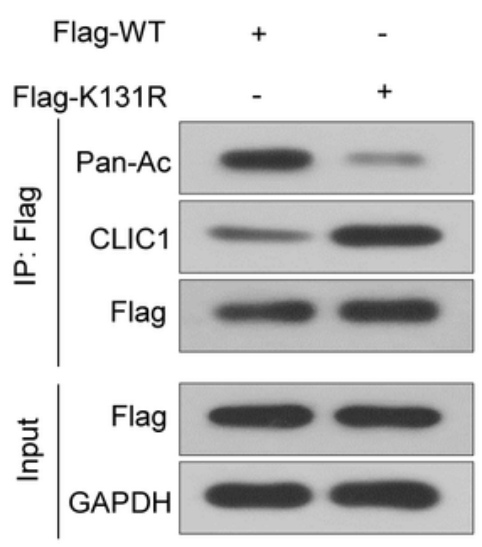

C

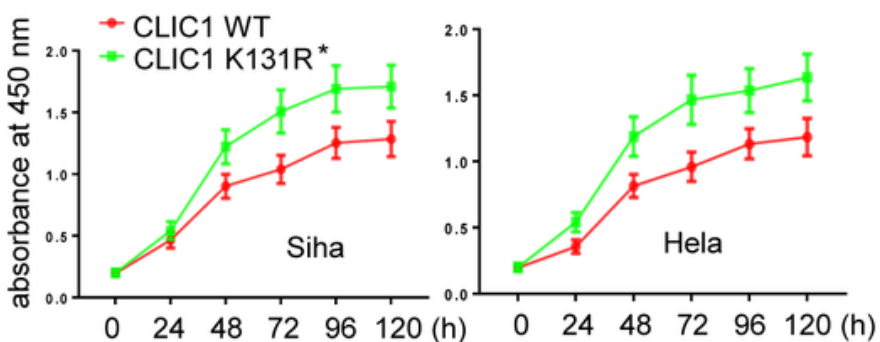

E
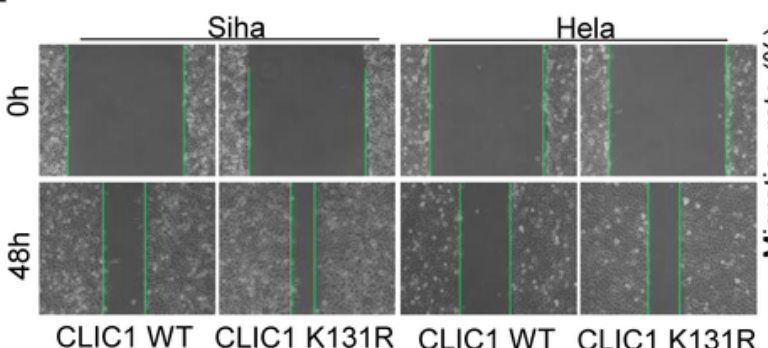

CLIC1 WT CLIC1 K131R
B

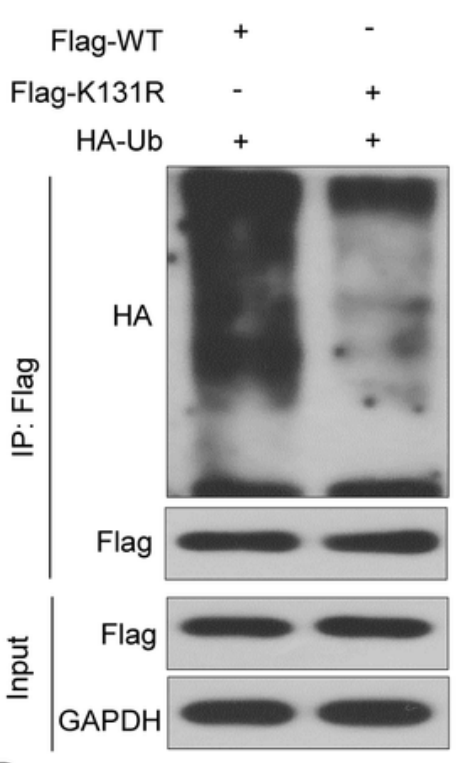

D

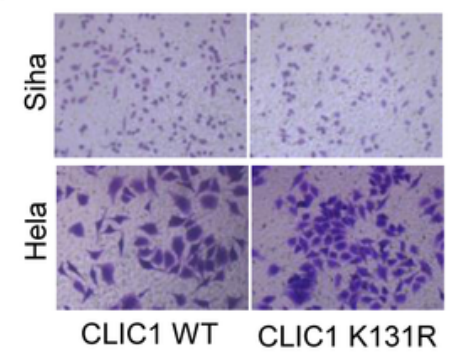

Hela

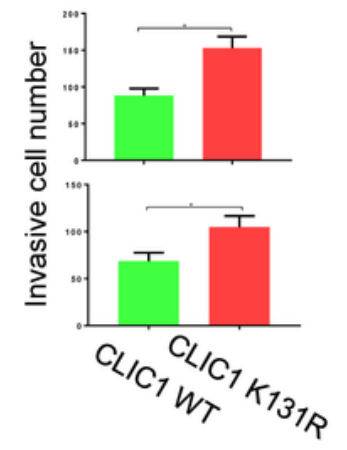

$\mathrm{F}$

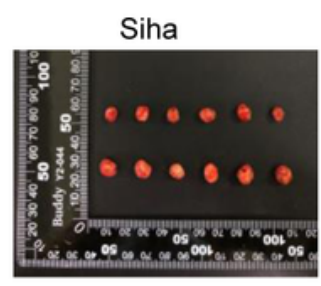

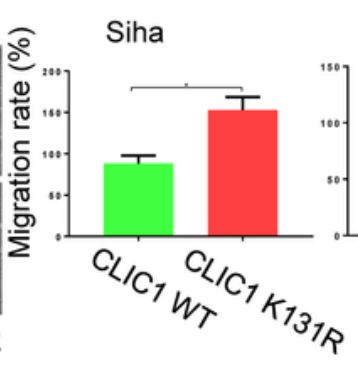

G

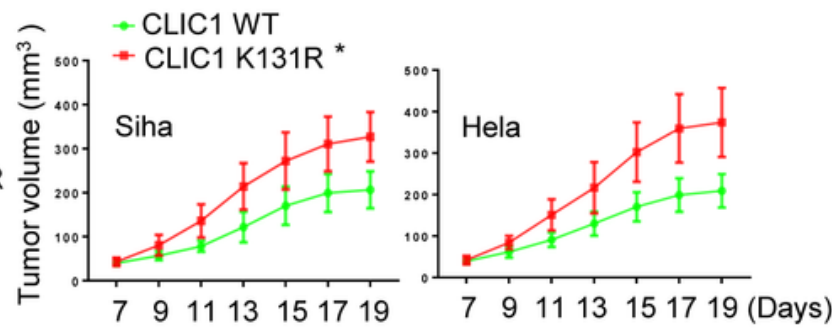

\section{Figure 7}

K131R mutant increased the stability of CLIC1 (A) Flag-tagged wild-type and K131R mutant CLIC1 were co-transfected into Hela cells, and acetylation of CLIC1 was determined by co-immunoprecipitation using Pan-Ac antibody. (B) Flag-tagged wild-type and K131R mutant CLIC1, and HA-tagged ubiquitin were cotransfected into Hela cells, The ubiquitylation of CLIC1 was assessed by co-immunoprecipitation and western blot assays. (C) Cell proliferation ability was analyzed by CCK-8 assay. (D) Cell invasive 
capability was evaluated by transwell assay (E) Cell migration ability was determined by scratch wound healing assay. Cells stably expressing wild type or K131R mutant CLIC1 were established and inoculated in the subcutaneous tissue of nude mice. (F) Photographs of xenograft tumors obtained from the nude mice. (G) Tumor growth curves. Error bars represent the SD of three independently prepared samples. $n=6$ per group for animal studies, $* \mathrm{P}<0.05$.

A
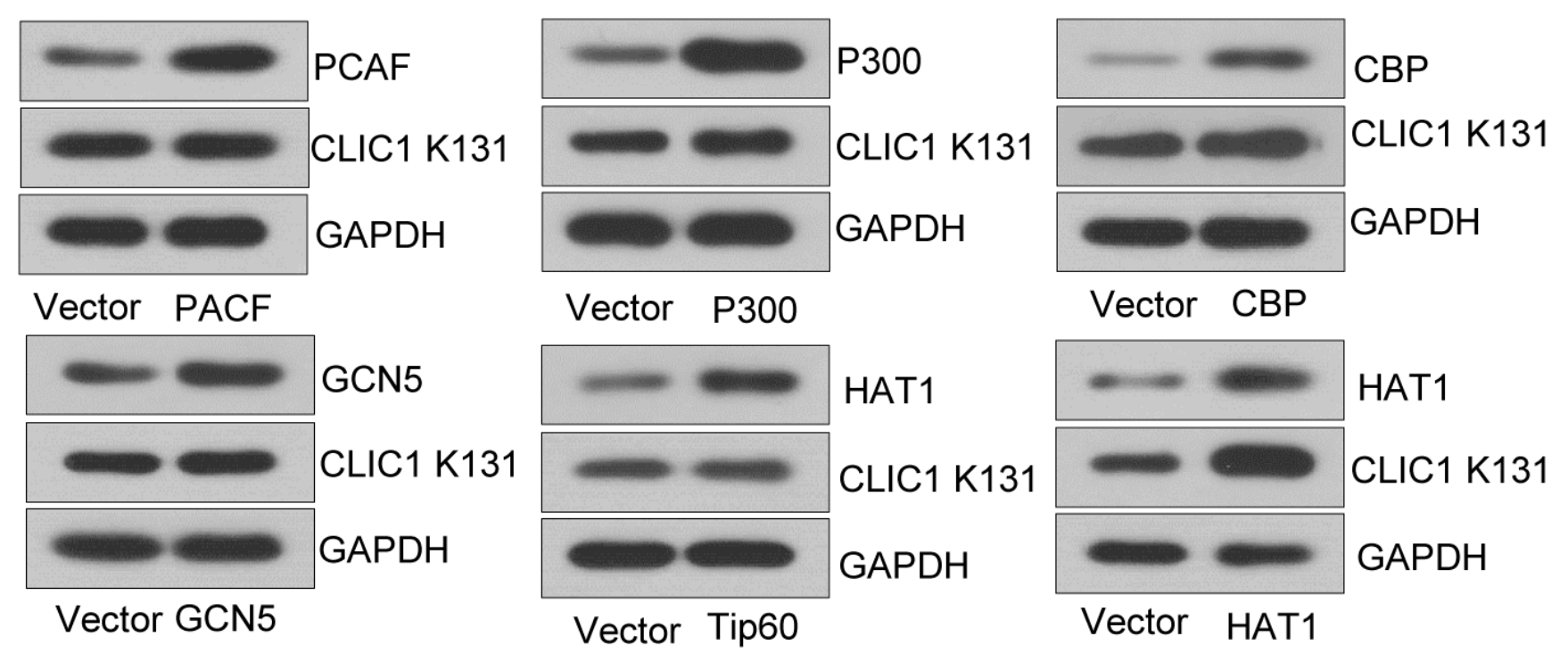

Vector CBP

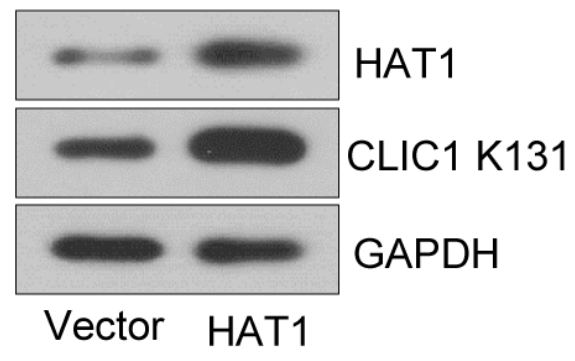

B
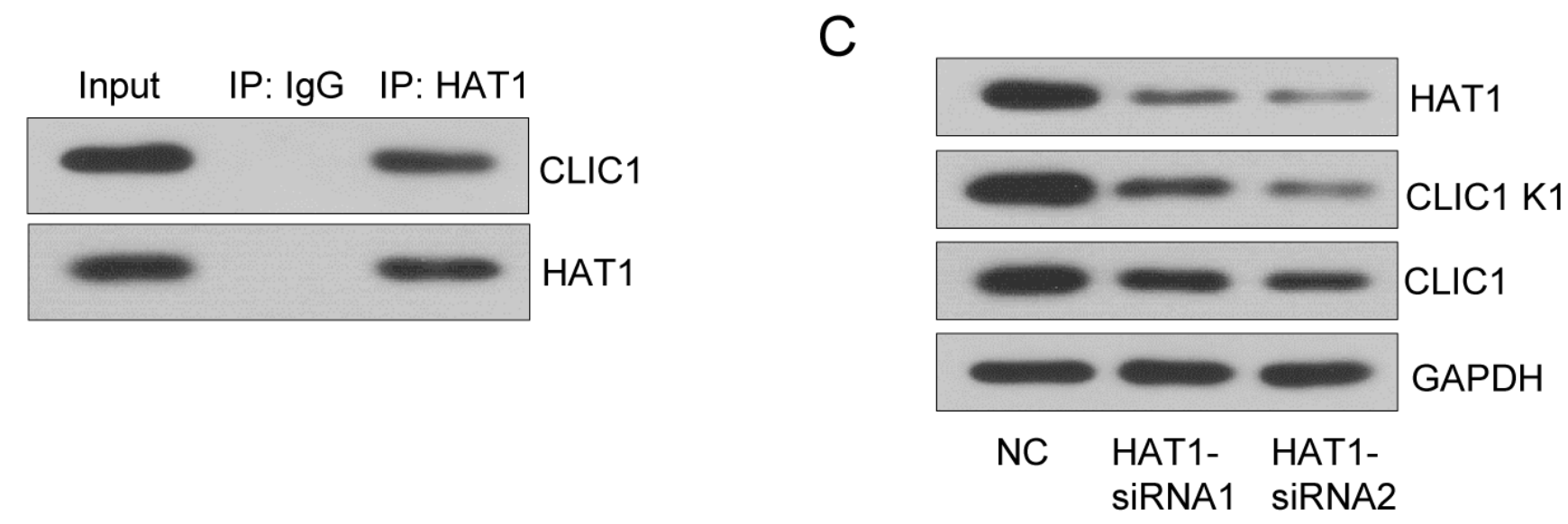

\section{Figure 8}

HAT-1 Acetylated CLIC1. (A) The expression levels of CLIC1 acetylation at K131 in Hela cells transfected with PCAF, P300, CBP, GCN5, Tip60, or HAT1 overexpressing plasmids were determined by western blot analysis. (B) In vivo interaction of HAT-1 with CLIC1 was assessed by co-IP and western blotting in Hela cells. (C) The expression levels of CLIC1 and CLIC1 acetylation at K131 in HAT1-silenced Hela cells were evaluated by western blot analysis. Error bars represent the SD of three independently prepared samples. $* \mathrm{P}<0.05$.

\section{Supplementary Files}


This is a list of supplementary files associated with this preprint. Click to download.

- Additionalfile1.tif

- Additionalfile1.tif 